\title{
Lysophosphatidylcholine aggravates contact hypersensitivity by promoting neutrophil infiltration and IL17 expression
}

\author{
Mi Hye Song ${ }^{1}$, Anupriya Gupta ${ }^{1}$, Hye One $\mathrm{Kim}^{2}$ \& Kwonik $\mathrm{Oh}^{1,3, *}$ \\ ${ }^{1}$ Department of Pathology, Hallym University College of Medicine, Chuncheon 24252, ${ }^{2}$ Department of Dermatology, Kangnam Sacred \\ Heart Hospital, Hallym University College of Medicine, Seoul 07441, ${ }^{3}$ Institute of Medical Science, Hallym University College of Medicine, \\ Chuncheon 24252, Korea
}

\begin{abstract}
Lysophosphatidylcholine (LPC) is a bioactive lysolipid known to contribute to the development of lung allergic diseases. However, it remains unknown whether LPC possesses proinflammatory properties in the skin as well. Here, we investigated this issue by injection of LPC into the murine contact hypersensitivity (CHS) model induced by 2,4-dinitrofluorobenzene (DNFB). LPC increased the expression of IL17, recruited more neutrophils, and eventually aggravated the CHS in the skins. Moreover, the effects of LPC diminished after neutralizing IL17 or depleting neutrophils. Mechanistically, LPC upregulated not only IL17 but also CXCL1 and CXCL2 in a G2A-dependent manner. Taken together, our study demonstrated that the upregulation of LPC could contribute to allergic skin inflammation by increasing IL17 expression and neutrophil recruitment via G2A receptor. [BMB Reports 2021; 54(4): 203-208]
\end{abstract}

\section{INTRODUCTION}

Contact hypersensitivity (CHS) is a representative type of T-cellmediated skin inflammation and also a murine model of human allergic contact dermatitis (1,2). As in other T-cell-mediated inflammatory models, CHS is divided into two distinct stages: sensitization and elicitation. During sensitization, haptens applied to abdominal skin are taken up by antigen-presenting cells, such as Langerhans cells and dermal dendritic cells, and bind to and modify self-antigens; then the modified self-antigens sensitize $\mathrm{CD}^{+}$and $\mathrm{CD}^{+} \mathrm{T}$ cells. After 5 to 7 days, we induced an elicitation response by application of the same haptens to the ear, to induce skin swelling; the extent of swelling was correlated with the severity (1). Although T-cell responses are predominant

*Corresponding author. Tel: +82-33-238-2564; Fax: +82-33-251-8250; E-mail: kwonik@hallym.ac.kr

https://doi.org/10.5483/BMBRep.2021.54.4.193

Received 8 September 2020, Revised 29 September 2020, Accepted 4 November 2020

Keywords: Cytokine, Inflammation, Lysophosphatidylcholine, Neutrophil, Skin in the pathology of $\mathrm{CHS}$, other immune cells, such as neutrophils (3), innate lymphoid cells (4), $\gamma \delta$ T cells $(5,6)$, NKT cells (7) and even B cells (8), can be involved.

LPC is a bioactive lysolipid produced by the cell-membrane metabolism by hydrolyzing phosphatidylcholine by phospholipase A2 (PLA2) in the Lands cycle (9). It can be divided into shortchain LPC or saturated/unsaturated LPC according to the acyl-chain length and degree of saturation (10). The levels of LPC can also be increased by lipid metabolism by means of the secretory PLA2 or the oxidative modification of lipoprotein phospholipids under inflammatory conditions $(11,12)$. In asthmatic patients (13) and animal models with lung injury (14), the concentrations of LPC were elevated in the lung fluids, accompanied by the increased activity of PLA2. Further study showed that the cell population responsible for the increase of LPC was the bronchial epithelial cells (15) and that LPC played a critical role in airway inflammation (16). LPC has various biological functions, including inflammatory responses, oxidative stress, and apoptosis (10), and the effects of LPC on immune cells are diverse, including increased bactericidal activity of neutrophils $(17,18)$, phagosome maturation of macrophages (19), integrin-mediated adhesion of eosinophils (20), activation of NKT cells $(21,22)$, and immune regulation (23). Altogether, these reports suggest that LPC can be upregulated under inflammatory conditions and play diverse roles, depending on environments.

Recently, it was reported that the proportion of short-chain LPC (C16 or C18) was increased significantly in atopic dermatitis, which is accompanied by downregulation of fatty-acid elongation enzymes, ELOVL3/ELOVL6 (24). Although these findings provide insight about how the expression of short-chain LPC is regulated, they did not show whether the 'increase of short-chain LPC' contributes to the development of skin inflammation.

Here, we examined the role of LPC in skin allergic inflammation in the DNFB-induced CHS model. We directly injected the short-chain LPC (C18:0) into the CHS mice and monitored the severity of the skin inflammation. Interestingly, LPC aggravated CHS by upregulating IL17 and CXCL1/2 and recruiting more neutrophils in a G2A-dependent manner. 


\section{RESULTS AND DISCUSSION}

\section{LPC aggravates DNFB-induced skin inflammation}

To investigate the roles of LPC in skin inflammation, we decided to use the DNFB-induced CHS model mimicking human allergic dermatitis. We applied a high dose of DNFB to the abdominal skin two times at d0 and d1 for sensitization and challenged the ear with a low dose of DNFB at d5 (Fig. 1A). On the next day, we measured the ear thickness every day as a readout of the skin inflammation. During the period of the experiment, we injected LPC into the test-group mice every day. We also prepared three different control groups: the first one we treated with acetone (vehicle of DNFB) only; the second we sensitized with acetone and challenged it with DNFB to assess the ear swelling induced by nonspecific irritation. Last, we sensitized the third group, challenged it with DNFB, but treated it with
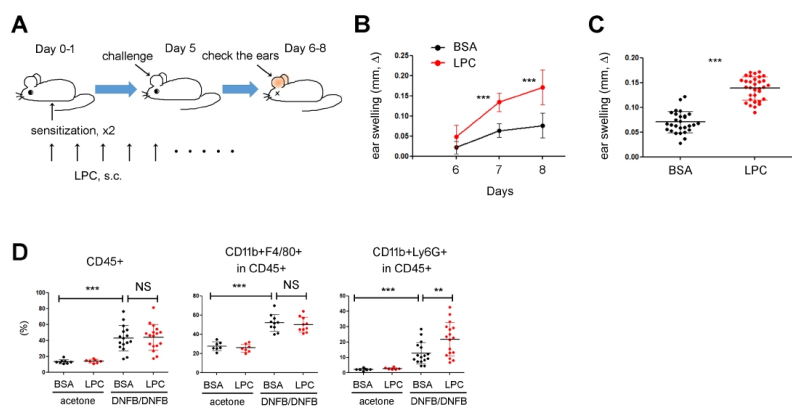

E
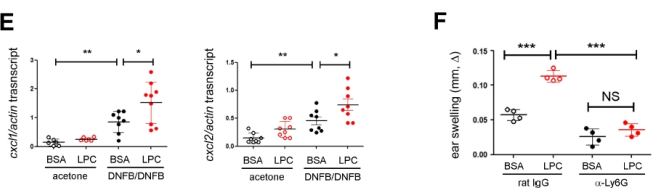

Fig. 1. LPC aggravated DNFB-induced skin inflammation. (A) Experimental design. We sensitized WT mice with $0.5 \%$ DNFB on d0 and $\mathrm{d} 1$, and then challenged them with $0.2 \%$ DNFB on $\mathrm{d} 5$. During the whole period, we injected LPC s.c. into the mice. The extent of CHS is shown as the increase of the ear thickness (ear swelling, $\Delta$ ), which we calculated by subtracting the ear thickness of the treated mice (DNFB sensitization and DNFB challenge) from that of the control mice (acetone sensitization and DNFB challenge: representing the nonspecific irritation). (B) Ear thickness results over time. We pooled data from five independent experiments. The difference of ear swelling between BSA and LPC was statistically significant on $d 7$ and d8. (C) Ear thickness results on $d 7$. We pooled data; each circle represents a single mouse. (D) FACS analysis of the ear skin. The percentages of hematopoietic cells (CD45 + left), macrophages $(\mathrm{CD} 11 \mathrm{~b}+\mathrm{F} 4 / 80+$, middle) and neutrophils (CD11b+Ly6G+, right) are shown. We pooled data; each circle represents a single mouse. (E) RT-qPCR analysis on the expressions of CXCL1 and CXCL2 in ear skin. We pooled data; each circle represents a single mouse. (F) We treated naïve mice with anti-Ly6G mAb to deplete neutrophils and then sensitized and challenged them with DNFB. The ear thickness results are shown. Data are representative of two independent experiments, and each circle represents a single mouse. Data are presented as the mean \pm SD. NS, not significant; $* P<0.05 ; * * P<0.01$; ***P $<0.001$.
BSA (vehicle of LPC). Ears treated with DNFB swelled significantly at $d 7-8$ and the swelling was aggravated by LPC (Fig. 1B and $1 \mathrm{C})$.

\section{More neutrophils were recruited into the ear skin by LPC}

Since the DNFB-induced skin inflammation is carried out by immune cells, we analyzed the phenotypes of immune cells in the skins by using flow cytometry. The percentage of total CD45 cells in the ear skins was increased significantly by DNFB, but was not changed further by LPC treatment (Fig. 1D, left). Next, we analyzed the adaptive immune-cell populations, such as $\mathrm{CD}^{+}$helper, $\mathrm{CD}^{+}{ }^{+}$cytotoxic, and Foxp3 ${ }^{+}$regulatory $\mathrm{T}$ cells, but did not find any significant difference between BSA and LPC (data not shown). Instead, the percentage of an innate cell population, like neutrophils $\left(\mathrm{CD} 11 \mathrm{~b}^{+} \mathrm{Ly} 6 \mathrm{G}^{+}\right.$; Fig. 1D, right), but not macrophages $\left(\mathrm{CD} 11 \mathrm{~b}^{+} \mathrm{F} 4 / 80^{+}\right.$; Fig. $1 \mathrm{D}$, middle), were increased by LPC. Since LPC had been reported to promote the activity of neutrophils, as in $\mathrm{H}_{2} \mathrm{O}_{2}$ production (17), we speculated that LPC could aggravate skin inflammations by increasing neutrophil infiltration. However, it was reported that LPC was not chemotactic for neutrophils in vitro (25), prompting us to analyze the expression of chemokines in the skin. Consistent with the assumption, the expressions of neutrophil chemokines, such as CXCL1 and CXCL2, were increased significantly by DNFB and even further after DNFB plus LPC treatment (Fig. 1E). Next, we depleted neutrophils by using anti-Ly6G monoclonal antibody $(\mathrm{mAb})$ and repeated the DNFB-induced CHS experiments (Supplementary Fig. 1). Interestingly, LPC failed to increase skin inflammation when neutrophilswere depleted (Fig. 1F), suggesting that LPC could control the recruitment of neutrophils in vivo by regulating chemokine expression, which aggravates skin inflammation.

\section{G2A is essential for the effects of LPC on CHS}

Although G2A (G2 Accumulation, an orphan G protein coupled receptor) is not a bona fide receptor of $\mathrm{LPC}$, many functions of LPC depend on G2A, which led us to investigate the roles of G2A in CHS. The expression of G2A increased significantly in DNFB-treated ears but was not changed further by LPC (Fig. $2 A)$. Next, we sought to investigate whether G2A contributes to LPC-induced chemokine expressions in the skin. The expression of CXCL2 of skin tissues was similar in both WT and G2A KO before LPC treatment. However, CXCL2 was significantly upregulated in WT but not in KO after LPC treatment (Fig. 2B, right). In the case of CXCL1, we also observed similar trends, although the difference between WT and G2A KOwas not statistically significant (Fig. 2B, left). Consistent with the chemokine expression results, in contrast to WT mice, the neutrophil infiltration into the ear skin was not increased by LPC treatment in G2A KO mice (Fig. 2C). We also monitored the extent of ear swelling and found that although the ears of both WT and G2A KO mice swelled after LPC treatment, the ears were swollen less in G2A KO mice (Fig. 2D). Interestingly, the ears of G2A KO mice swelled less than did those of WT even 
A

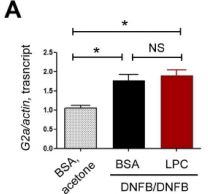

C
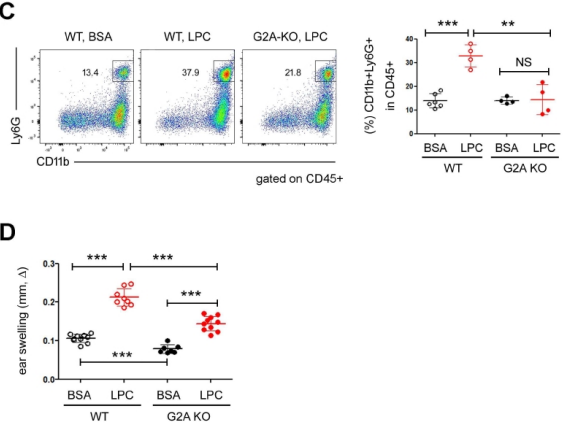

Fig. 2. G2A mediated the effects of LPC on neutrophils. (A) RT-qPCR analysis on the expressions of G2A. We subjected the whole ear skin tissues to RNA extraction and used it for RT-qPCR study. Data are representative of two independent experiments. (B) RT-qPCR analysis on the expressions of CXCL1 and CXCL2 in ear skin. We pooled data; each circle represents a single mouse. (C) FACS analysis of the ear skins of WT and G2A KO mice in DNFB-induced $\mathrm{CHS}$. The percentages of neutrophils $(\mathrm{CD} 11 \mathrm{~b}+\mathrm{Ly} 6 \mathrm{G}+)$ in $\mathrm{CD} 45+$ population are shown. Statistical analysis is shown in the right panel. We pooled data; each circle represents a single mouse. (D) Ear thickness results of WT and G2A KO mice. Statistical analysis is shown. We pooled data; each circle represents a single mouse. Data are presented as the mean \pm SD. NS, not significant; $* P<0.05 ; * * P<0.01$; $* * * \mathrm{P}<0.001$.

when stimulated with DNFB plus BSA (Fig. 2D), implying that G2A might play non-redundant roles in the DNFB-induced skin inflammation quite apart from exogenous LPC stimulation (26). Altogether, our study demonstrated that LPC aggravated the DNFB-induced skin inflammation in a G2A-dependent manner.

\section{IL17 was upregulated by LPC treatment}

Next, we sought to investigate the expressions of IFN- $\gamma$ and IL17 as signature cytokines of T-cell-mediated inflammations. Interestingly, the percentage of IL17-expressing cells increased in LPC-treated ear skins (Fig. 3A). We also observed similar results in RT-qPCR analysis (Fig. 3B). The subsequent FACS analysis revealed that the majority of IL17-expressing cells were TCR $\gamma \delta$ cells. In contrast, $\mathrm{TCR}^{+}$cellswere the major cellular sources of IFN- $\gamma$ (Supplementary Fig. 2A). To check whether LPC is directly involved in the regulation of IL17 expression in T cells, we sought to culture naïve $\mathrm{CD} 4^{+} \mathrm{T}$ and $\mathrm{TCR} \delta^{+}$cells in the presence of LPC under T helper type 17 (TH17) conditions and checked the expression of IL17. Unexpectedly, IL17 was not upregulated by LPC (data not shown), which caused concern that there might be another type of immune cell, such as innate lymphoid cells, not T cells, that produce IL17 in respond to LPC. Therefore, we repeated the
A
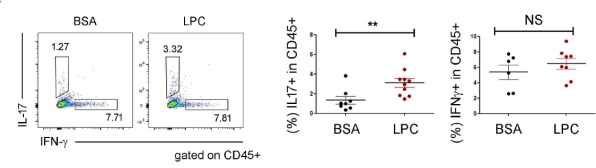

B

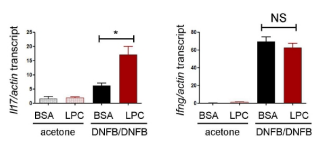

Fig. 3. LPC upregulated IL17. (A) The FACS analysis of IL17 and IFN- $\gamma$ in CD45 + cells of the ears. Statistical analysis is shown in the right. We pooled data; each circle represents a single mouse. (B) RT-qPCR analysis on the expressions of IL17 and IFN- $\gamma$. We subjected the whole skin tissues to RNA extraction and used them for RT-qPCR study. Data are presented as the mean \pm SD. NS, not significant; $* P<0.05 ; * * P<0.01 ; * * * P<0.001$.

CHS experiments in lymphocyte-deficient RAG-1 KO mice to confirm the role of T cells. RAG-1 KO mice developed the muchmilder CHS, as shown previously (27), and the ear swelling of RAG-1 KO mice was not increased significantly by LPC (Supplementary Fig. 2B). Moreover, the percentage of IL17-expressing cells was pretty low in RAG-1 KO mice treated with DNFB plus BSA andwas not increased by LPC (Supplementary Fig. 2C), indicating that LPC upregulated IL17 indirectly in T cells.

\section{The effects of LPC on CHS is mediated by IL17}

IL17 is a signature cytokine in type 3 inflammation where neutrophils play an important role. We realized in this study that LPC upregulated IL17 (Fig. 3A), recruited neutrophils more efficiently (Fig. 1D), and exacerbated DNFB-induced skin inflammation (Fig. 1C). These findings led us to assume that LPC could recruit neutrophils by using IL17, which aggravated skin inflammation. To confirm the above hypothesis, we neutralized IL17 cytokine by using anti-IL17 mAb and did the CHS experiments. In mice treated with DNFB plus BSA, IL17 neutralization reduced the ear swelling slightly (Fig. 4A) but did not prevent the neutrophil accumulation (Fig. 4B and 4C). However, the effects of LPC on skin inflammation were dramatically reduced by IL17 neutralization. The ears treated with anti-IL17 mAb did not swell as much as did those treated with isotype control $\mathrm{mAb}$ in the presence of LPC (Fig. 4A). Furthermore, IL17 neutralization abolished the neutrophil recruitment (Fig $4 \mathrm{~B}$ and $4 \mathrm{C}$ ) and the upregulation of CXCL1/2 (Fig. 4D) induced by LPC, implying that the inflammatory effects of LPC depend on IL17.

Last, we investigated whether the upregulation of IL17 induced by LPC also depended on G2A and found that the expression of IL17 did not increase in G2A KO mice as much as in WT in the presence of LPC stimulation (Fig. 4E).

In this study, we investigated the effect of LPC in a gain-offunction approach and found that LPC upregulated CXCL1 and CXCL2 (Fig. 1E), recruited neutrophils (Fig. 1D), and aggravated DNFB-induced CHS (Fig. 1C). Moreover, once neutrophils were depleted, LPC did not cause the ear swelling anymore (Fig. 
A

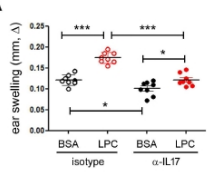

B
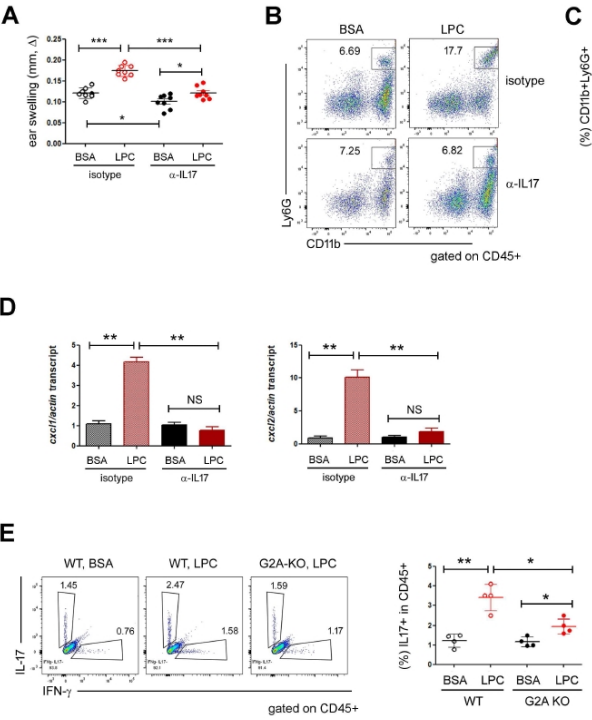

Fig. 4. LPC exacerbated CHS in an IL17-dependent manner. (A) Ear thickness results after anti-IL17 mAb treatment. We pooled data; each circle represents a single mouse. $(B, C)$ FACS analysis of the ear skin after anti-IL17 mAb treatment. The percentages of neutrophils (CD11b+ $\mathrm{Ly} 6 \mathrm{G}+$ ) in $\mathrm{CD} 45+$ population are shown in (B). (D) RT-qPCR analysis on the expressions of CXCL1 (left) and CXCL2 (right). We subjected the whole skin tissues to RNA extraction and used them for RT-qPCR study. Data are representative of two independent experiments. (E) FACS analysis on the expressions of IL17 and IFN- $\gamma$ in the WT and $\mathrm{G} 2 \mathrm{~A} \mathrm{KO}$ skins. The frequency of the cytokine-expressing cells in the CD45+ population is shown. Statistical analysis is shown in the right panel. Data are representative of two independent experiments and each circle represents one mouse. Data are presented as the mean \pm SD. NS, not significant; $* P<0.05 ; * * P<0.01 ; * * * P<$ 0.001 .

1F). These findings clearly indicated that neutrophils recruited by LPC and CXCL1/2 exacerbated CHS. Then, how was CXCL1/2 upregulated by LPC? Based on our finding that IL17was upregulated by LPC (Fig. 3) and the previous reports that IL17 drives neutrophil infiltration by inducing the expression of neutrophilattracting chemokines such as CXCL1/2 (28-31), we hypothesized that LPC upregulated IL17, which subsequently increased the CXCL1/2 expressions and neutrophil infiltration. This hypothesiswas supported by the findings of our IL17 neutralization experiments (Fig. 4). However, we failed to identify the detailed mechanisms by which LPC upregulated IL17, which need further study.

The putative LPC receptor is the G protein coupled receptors, $\mathrm{G} 2 \mathrm{~A}$. Although G2A KO mice developed a late-onset autoimmune disease that looked like Systemic Lupus Erythematosus (32), recent studies have shown evidence that G2A can work as both pro- and anti-inflammatory mediators $(26,33,34)$. Here, we demonstrated that LPC exacerbated CHS in a G2A-dependent manner (Fig. 2D), suggesting the proinflammatory roles of LPC and G2A in skin inflammations. Particularly, it was intriguing that $\mathrm{G} 2 \mathrm{~A} \mathrm{KO}$ mice developed less-severe $\mathrm{CHS}$ even in the absence of LPC treatment. Given that diverse G2A ligands including LPC and oxidized fatty acids (35) are available in skin, these findings imply the importance of G2A and its lipid ligands in skin homeostasis.

In conclusion, we demonstrated that the upregulation of LPC could exacerbate allergic skin inflammation by increasing IL17 expression and neutrophil recruitment via G2A receptor. Further study on LPC and G2A would help our understanding of the roles of lipid metabolites in skin immunology.

\section{MATERIALS AND METHODS}

\section{Mice}

We purchased WT C57BL/6 mice from Koatech (Pyeongtaek, Korea). The G2A knockout (KO) mice (36) on the C57BL/6 background, as we described previously, we received from Dr. DK Song (Hallym University). We did all animal experiments in accordance with guidelines and approval of the International Animal Care and Use Committees of Hallym University (Hallym 2018-9, 2019-18).

\section{Sensitization and elicitation of CHS}

We did the induction of CHS in mice as described previously $(37,38)$. The extent of CHS was shown as the increase of the ear thickness (ear swelling), which we calculated by subtracting the ear thickness of the treated mice from that of the control mice (mice challenged with DNFB (Sigma Korea, Seoul, Korea) without sensitization), which we measured every $24 \mathrm{~h}$ after challenging them using a micrometer (Mitutoyo, Kanagawa, Japan). We injected LPC (18:0 Lyso PC, 1-stearoyl-2-hydroxy-snglycero-3-phosphocholine, Avanti Polar Lipids, Alabaster, AL) or $2 \%$ BSA (vehicle of LPC, Sigma) subcutaneously for the whole period of the experiments.

\section{Tissue preparation and flow cytometry}

We removed ears, split them in half, and cut them into small pieces. We treated skin tissues in RPMI media containing 0.1 $\mathrm{mg} / \mathrm{ml}$ DNase I and $0.1 \mathrm{mg} / \mathrm{ml}$ Liberase TL (Sigma) for $2 \mathrm{~h}$ at $37^{\circ} \mathrm{C}$. We filtered digested tissues with a $70-\mu \mathrm{m}$ cell strainer (SPL, Seoul, Korea). For cytokine analysis, we cultured cells for $4 \mathrm{~h}$ in the presence of PMA/ionomycin plus monensin (BD Biosciences, San Jose, CA) before intracellular cytokine staining unless otherwise specified. We acquired data by means of FACS Canto-II (BD Biosciences) and analyzed the data with Flowjo software (BD Biosciences).

\section{Quantitative RT-PCR (RT-qPCR)}

We isolated RNA using the RNeasy Mini kit (Qiagen, Germantown, MD) or Trizol (Thermo Fisher Scientific Korea, Seoul, Korea), and reverse-transcribed it into cDNA using QuantiTect Reverse Transcription kit (Qiagen). We normalized all data to actin. We checked non-specific amplification by the use of melting curves and agarose gel electrophoresis (39). The sequences of primers 
(Genotech, Daejon, Korea) are as follows. Il17a forward, 5'-AC TACCTCAACCGTTCCACGTC-3'; II17a reverse, 5'-ATGTGGTGG TCCAGCTTTCC-3'; Ifng forward, $5^{\prime}$-GATGCATTCATGAGTATT GCCAAGT-3'; Ifng reverse, 5'-GTGGACCACTCGGATGAGCTC3'; Cxcl1 forward, 5'-TGAGCTGCGCTGTCAGTGCCT-3'; Cxcl1 reverse, 5'-AGAAGCCAGCGTTCACCAGA-3'; Cxcl2 forward, 5'GAGCTTGAGTGTGACGCCCCCAGG-3'; Cxcl2 reverse, 5'-GTT AGCCTTGCCTTTGTTCAGTATC-3'; G2a forward, 5'-AAGTGT CCAGAATCCACACAGGGT-3'; G2a reverse, 5'-AGTAAACCTA GCTTCGCTGGCTGT-3'; actin forward, 5'-CATCCGTAAAGACC TCTATGCCAAC-3'; actin reverse, 5'-ATGGAGCCACCGATCCA CA-3'.

\section{Statistical analyses}

We used a two-tailed, unpaired, student's t-test to calculate the statistical significance of differences between groups unless specified. We represented $\mathrm{P}$ values as follows: ${ }^{* * *} \mathrm{P}<0.001$; ${ }^{*} * \mathrm{P}<0.01$; $* \mathrm{P}<0.05$, whereas we used NS, not significant, to denote $\mathrm{P}>0.05$. Error bars indicate s.d.

\section{ACKNOWLEDGEMENTS}

This work was supported by NRF of Korea (2018R1D1A1B070 4642813), Hallym University (H20160646) and Korea Healthcare R\&D project (HI17C0597).

\section{CONFLICTS OF INTEREST}

The authors have no conflicting interests.

\section{REFERENCES}

1. Kaplan DH, Igyarto BZ and Gaspari AA (2012) Early immune events in the induction of allergic contact dermatitis. Nat Rev Immunol 12, 114-124

2. Honda T, Egawa G, Grabbe S and Kabashima K (2013) Update of immune events in the murine contact hypersensitivity model: toward the understanding of allergic contact dermatitis. J Invest Dermatol 133, 303-315

3. Weber FC, Nemeth T, Csepregi JZ et al (2015) Neutrophils are required for both the sensitization and elicitation phase of contact hypersensitivity. J Exp Med 212, 15-22

4. Rafei-Shamsabadi DA, van de Poel S, Dorn B et al (2018) Lack of type 2 innate lymphoid cells promotes a type I-driven increased immune response in contact hypersensitivity. J Invest Dermatol 138, 1962-1972

5. Jiang X, Park CO, Geddes Sweeney J, Yoo MJ, Gaide O and Kupper TS (2017) Dermal gammadelta T cells do not freely re-circulate out of skin and produce IL-17 to promote neutrophil infiltration during primary contact hypersensitivity. PLoS One 12, e0169397

6. Nielsen MM, Lovato P, MacLeod AS et al (2014) IL-1betadependent activation of dendritic epidermal $\mathrm{T}$ cells in contact hypersensitivity. J Immunol 192, 2975-2983

7. Shimizuhira C, Otsuka A, Honda T et al (2014) Natural killer T cells are essential for the development of contact hypersensitivity in BALB/c mice. J Invest Dermatol 134, 2709-2718

8. Kim HS, Lee MB, Lee D et al (2019) The regulatory B cell-mediated peripheral tolerance maintained by mast cell IL-5 suppresses oxazolone-induced contact hypersensitivity. Sci Adv 5, eaav8152

9. Law SH, Chan ML, Marathe GK, Parveen F, Chen $\mathrm{CH}$ and Ke LY (2019) An updated review of lysophosphatidylcholine metabolism in human diseases. Int J Mol Sci 20, 1149

10. Liu P, Zhu W, Chen $C$ et al (2020) The mechanisms of lysophosphatidylcholine in the development of diseases. Life Sci 247, 117443

11. Fuchs B, Schiller J, Wagner $U$, Hantzschel $H$ and Arnold K (2005) The phosphatidylcholine/lysophosphatidylcholine ratio in human plasma is an indicator of the severity of rheumatoid arthritis: investigations by 31P NMR and MALDITOF MS. Clin Biochem 38, 925-933

12. Kabarowski JH (2009) G2A and LPC: regulatory functions in immunity. Prostaglandins Other Lipid Mediat 89, 73-81

13. Yoder M, Zhuge Y, Yuan Y et al (2014) Bioactive lysophosphatidylcholine 16:0 and 18:0 are elevated in lungs of asthmatic subjects. Allergy Asthma Immunol Res 6, 61-65

14. Arbibe L, Koumanov K, Vial D et al (1998) Generation of lyso-phospholipids from surfactant in acute lung injury is mediated by type-II phospholipase A2 and inhibited by a direct surfactant protein A-phospholipase A2 protein interaction. J Clin Invest 102, 1152-1160

15. Zhuge Y, Yuan Y, van Breemen R et al (2014) Stimulated bronchial epithelial cells release bioactive lysophosphatidylcholine 16:0, 18:0, and 18:1. Allergy Asthma Immunol Res 6, 66-74

16. Bansal P, Gaur SN and Arora N (2016) Lysophosphatidylcholine plays critical role in allergic airway disease manifestation. Sci Rep 6, 27430

17. Yan JJ, Jung JS, Lee JE et al (2004) Therapeutic effects of lysophosphatidylcholine in experimental sepsis. Nat Med 10, 161-167

18. Hong CW, Kim TK, Ham HY et al (2010) Lysophosphatidylcholine increases neutrophil bactericidal activity by increasement of azurophil granule-phagosome fusion via glycine. GlyR alpha 2/TRPM2/p38 MAPK signaling. J Immunol 184, 4401-4413

19. Lee HJ, Ko HJ, Song DK and Jung YJ (2018) Lysophosphatidylcholine promotes phagosome maturation and regulates inflammatory mediator production by means of the protein kinase A-phosphatidylinositol 3 kinase-p38 mitogenactivated protein kinase signaling pathway during mycobacterium tuberculosis infection in mouse macrophages. Front Immunol 9, 920

20. Zhu X, Learoyd J, Butt S et al (2007) Regulation of eosinophil adhesion by lysophosphatidylcholine via a non-storeoperated Ca2 + channel. Am J Respir Cell Mol Biol 36, 585-593

21. Fox LM, Cox DG, Lockridge JL et al (2009) Recognition of lyso-phospholipids by human natural killer T lymphocytes. PLoS Biol 7, e1000228

22. Maricic I, Girardi E, Zajonc DM and Kumar V (2014) Recognition of lysophosphatidylcholine by type II NKT cells and protection from an inflammatory liver disease. J Immunol 193, 4580-4589 
23. Hasegawa H, Lei J, Matsumoto T, Onishi S, Suemori K and Yasukawa M (2011) Lysophosphatidylcholine increases the suppressive function of human naturally occurring regulatory T cells by means of TGF-beta production. Biochem Biophys Res Commun 415, 526-531

24. Berdyshev E, Goleva E, Bronova I et al (2018) Lipid abnormalities in atopic skin are driven by type 2 cytokines. $\mathrm{JCl}$ Insight 3, e98006

25. Quinn MT, Parthasarathy S and Steinberg D (1988) Lysophosphatidylcholine: a chemotactic factor for human monocytes and its potential role in atherogenesis. Proc Natl Acad Sci U S A 85, 2805-2809

26. Hattori T, Obinata H, Ogawa A et al (2008) G2A plays proinflammatory roles in human keratinocytes under oxidative stress as a receptor for 9-hydroxyoctadecadienoic acid. J Invest Dermatol 128, 1123-1133

27. Paust S, Gill HS, Wang BZ et al (2010) Critical role for the chemokine receptor CXCR6 in NK cell-mediated antigenspecific memory of haptens and viruses. Nat Immunol 11, 1127-1135

28. Ye P, Rodriguez FH, Kanaly S et al (2001) Requirement of interleukin 17 receptor signaling for lung CXC chemokine and granulocyte colony-stimulating factor expression, neutrophil recruitment, and host defense. J Exp Med 194, 519-527

29. Miyamoto M, Prause O, Sjostrand M, Laan M, Lotvall J and Linden A (2003) Endogenous IL-17 as a mediator of neutrophil recruitment caused by endotoxin exposure in mouse airways. J Immunol 170, 4665-4672

30. Sun D, Novotny M, Bulek K, Liu C, Li X and Hamilton T (2011) Treatment with IL-17 prolongs the half-life of chemokine CXCL1 mRNA via the adaptor TRAF5 and the splicingregulatory factor SF2 (ASF). Nat Immunol 12, 853-860

31. Geha M, Tsokos MG, Bosse RE et al (2017) IL-17A pro- duced by innate lymphoid cells is essential for intestinal ischemia-reperfusion injury. J Immunol 199, 2921-2929

32. Le LQ, Kabarowski JH, Weng $Z$ et al (2001) Mice lacking the orphan $\mathrm{G}$ protein-coupled receptor G2A develop a late-onset autoimmune syndrome. Immunity 14, 561-571

33. Kern K, Schafer SMG, Cohnen J et al (2018) The G2A receptor controls polarization of macrophage by determining their localization within the inflamed tissue. Front Immunol 9, 2261

34. Frasch SC, McNamee EN, Kominsky D et al (2016) G2A signaling dampens colitic inflammation via production of IFN-gamma. J Immunol 197, 1425-1434

35. Obinata $\mathrm{H}$ and Izumi $\mathrm{T}$ (2009) $\mathrm{G} 2 \mathrm{~A}$ as a receptor for oxidized free fatty acids. Prostaglandins Other Lipid Mediat 89, 6672

36. Li HM, Jang JH, Jung JS et al (2019) G2A protects mice against sepsis by modulating kupffer cell activation: cooperativity with adenosine receptor $2 \mathrm{~b}$. J Immunol 202, 527-538

37. Engeman T, Gorbachev AV, Kish DD and Fairchild RL (2004) The intensity of neutrophil infiltration controls the number of antigen-primed CD8 T cells recruited into cutaneous antigen challenge sites. J Leukoc Biol 76, 941-949

38. He D, Wu L, Kim HK, Li H, Elmets CA and Xu H (2009) IL-17 and IFN-gamma mediate the elicitation of contact hypersensitivity responses by different mechanisms and both are required for optimal responses. J Immunol 183, 1463-1470

39. Kim $\mathrm{G}$, Jeong $\mathrm{H}$, Youn $\mathrm{H}$ et al (2020) Anti-inflammatory mechanisms of suppressors of cytokine signaling target ROS via NRF-2/thioredoxin induction and inflammasome activation in macrophages. BMB Rep 53, 640-645 